Mathematical Research Letters 10, 671-684 (2003)

\title{
ELLIPTIC SELBERG INTEGRALS AND CONFORMAL BLOCKS
}

\author{
G. Felder, L. Stevens, and A. Varchenko
}

Abstract. We present an elliptic version of Selberg's integral formula.

\section{Introduction}

The Selberg integral is the integral

$$
B_{p}(\alpha, \beta, \gamma)=\int_{\Delta_{p}} \prod_{j=1}^{p} t_{j}{ }^{\alpha-1}\left(1-t_{j}\right)^{\beta-1} \prod_{0 \leq j<k \leq p}\left(t_{j}-t_{k}\right)^{2 \gamma} d t_{1} \ldots d t_{p},
$$

where $\Delta_{p}=\left\{t \in \mathbb{R}^{p} \mid 0 \leq t_{p} \leq \cdots \leq t_{1} \leq 1\right\}$. The Selberg integral is a generalization of the beta function. It can be calculated explicitly,

$$
B_{p}(\alpha, \beta, \gamma)=\frac{1}{p !} \prod_{j=0}^{p-1} \frac{\Gamma(1+\gamma+j \gamma) \Gamma(\alpha+j \gamma) \Gamma(\beta+j \gamma)}{\Gamma(1+\gamma) \Gamma(\alpha+\beta+(p+j-1) \gamma)} .
$$

The Selberg integral has many applications, see [A1, A2, As, D, DF1, DF2, M, S]. In this paper, we present elliptic versions of the Selberg integral.

\section{Conformal Blocks on the Torus}

Let $\tau \in \mathbb{C}$ be such that $\operatorname{Im} \tau>0$. Let $\kappa$ and $p$ be non-negative integers satisfying $\kappa \geq 2 p+2$. The KZB-heat equation is the partial differential equation

$$
2 \pi i \kappa \frac{\partial u}{\partial \tau}(\lambda, \tau)=\frac{\partial^{2} u}{\partial \lambda^{2}}(\lambda, \tau)+p(p+1) \rho^{\prime}(\lambda, \tau) u(\lambda, \tau) .
$$

Here, the prime denotes the derivative with respect to the first argument, and $\rho$ is defined in terms of the first Jacobi theta function,

$$
\begin{gathered}
\vartheta_{1}(\lambda, \tau)=2 q^{\frac{1}{8}} \sin (\pi \lambda) \prod_{j=1}^{\infty}\left(1-q^{j} e^{2 \pi i \lambda}\right)\left(1-q^{j} e^{-2 \pi i \lambda}\right)\left(1-q^{j}\right), \\
\rho(\lambda, \tau)=\frac{\vartheta_{1}^{\prime}(\lambda, \tau)}{\vartheta_{1}(\lambda, \tau)},
\end{gathered}
$$

where $q=e^{2 \pi i \tau}$. Holomorphic solutions of the KZB-heat equation with the properties,

Received October 2, 2002.

Supported in part by NSF grant DMS-9801582. 
(i) $u(\lambda+2, \tau)=u(\lambda, \tau)$,

(ii) $u(\lambda+2 \tau, \tau)=e^{-2 \pi i \kappa(\lambda+\tau)} u(\lambda, \tau)$,

(iii) $u(-\lambda, \tau)=(-1)^{p+1} u(\lambda, \tau)$,

(iv) $u(\lambda, \tau)=\mathcal{O}\left((\lambda-m-n \tau)^{p+1}\right)$ as $\lambda \rightarrow m+n \tau$ for any $m, n \in \mathbb{Z}$

are called conformal blocks (or elliptic hypergeometric functions) associated with the family of elliptic curves $\mathbb{C} / \mathbb{Z}+\tau \mathbb{Z}$ with the marked point $z=0$ and the irreducible $s l_{2}$ representation of dimension $2 p+1$. It is known that the space of conformal blocks has dimension $\kappa-2 p-1$.

\section{Integral Representations of Conformal Blocks}

Introduce special functions

$$
\sigma_{\lambda}(t, \tau)=\frac{\vartheta_{1}(\lambda-t, \tau) \vartheta_{1}^{\prime}(0, \tau)}{\vartheta_{1}(\lambda, \tau) \vartheta_{1}(t, \tau)}, \quad E(t, \tau)=\frac{\vartheta_{1}(t, \tau)}{\vartheta_{1}^{\prime}(0, \tau)}
$$

Consider the theta functions

$$
\theta_{\kappa, n}(\lambda, \tau)=\sum_{j \in \mathbb{Z}} e^{2 \pi i \kappa\left(j+\frac{n}{2 \kappa}\right)^{2} \tau+2 \pi i \kappa\left(j+\frac{n}{2 \kappa}\right) \lambda}, \quad n \in \mathbb{Z} / 2 \kappa \mathbb{Z} .
$$

They form a basis of the space of theta functions of level $\kappa$. They satisfy the equations

$$
\theta_{\kappa, n}(\lambda+1, \tau)=(-1)^{n} \theta_{\kappa, n}(\lambda, \tau), \quad \theta_{\kappa, n}(\lambda+\tau, \tau)=e^{-\pi i \kappa\left(\lambda+\frac{\tau}{2}\right)} \theta_{\kappa, n+\kappa}(\lambda, \tau)
$$

and have the modular properties

$$
\begin{gathered}
\theta_{\kappa, n}(\lambda, \tau+1)=e^{\pi i \frac{n^{2}}{2 \kappa}} \theta_{\kappa, n}(\lambda, \tau), \\
\theta_{\kappa, n}\left(\frac{\lambda}{\tau},-\frac{1}{\tau}\right)=\sqrt{-\frac{i \tau}{2 \kappa}} e^{\pi i \kappa \frac{\lambda^{2}}{2 \tau}} \sum_{m=0}^{2 \kappa-1} e^{-\pi i \frac{m n}{\kappa}} \theta_{\kappa, m}(\lambda, \tau),
\end{gathered}
$$

where $|\arg (-i \tau)|<\pi / 2$. Let $\theta_{\kappa, n}^{s}$ denote the symmetrization of $\theta_{\kappa, n}$ with respect to $\lambda, \theta_{\kappa, n}^{s}(\lambda, \tau)=\theta_{\kappa, n}(\lambda, \tau)+\theta_{\kappa, n}(-\lambda, \tau)$.

Define $u_{\kappa, n}$ by

$$
u_{\kappa, n}(\lambda, \tau)=u_{p, \kappa, n}(\lambda, \tau)=J_{p, \kappa, n}(\lambda, \tau)+(-1)^{p+1} J_{p, \kappa, n}(-\lambda, \tau),
$$

where

$$
\begin{aligned}
J_{p, \kappa, n}(\lambda, \tau)=\int_{\Delta_{p}} \prod_{j=1}^{p} E\left(t_{j}, \tau\right)^{-\frac{2 p}{\kappa}} \prod_{1 \leq j<k \leq p} E\left(t_{j}-t_{k}, \tau\right)^{\frac{2}{\kappa}} \times \\
\prod_{j=1}^{p} \sigma_{\lambda}\left(t_{j}, \tau\right) \theta_{\kappa, n}\left(\lambda+\frac{2}{\kappa} \sum_{j=1}^{p} t_{j}, \tau\right) d t_{1} \ldots d t_{p} .
\end{aligned}
$$


The branch of the logarithm is chosen in such a way that $\arg (E(t, \tau)) \rightarrow 0$ as $t \rightarrow 0^{+}$, and the integral is understood as the analytic continuation from the region where all of the exponents in the integrand have positive real parts.

Theorem 3.1. [FV1] For all $n$, the integrals $u_{\kappa, n}(\lambda, \tau)$ are solutions of the KZB-heat equation having the properties (i)-(iv).

Theorem 3.2. [FSV2] We have

(a) $u_{\kappa, n}=u_{\kappa, n+2 \kappa}$ and $u_{\kappa, n}=-e^{2 \pi i p n / \kappa} u_{\kappa,-n}$.

(b) The set $\left\{u_{\kappa, n}(\lambda, \tau) \mid n=p+1, \ldots, \kappa-p-1\right\}$ is a basis for the space of conformal blocks. The integrals $u_{\kappa, n}$ are identically zero for all other values of $n$ in the interval from 0 to $\kappa$.

\section{Transformations acting on the space of conformal blocks}

Introduce four transformations $A, B, T$, and $S$ defined by

$$
\begin{gathered}
A u(\lambda, \tau)=u(\lambda+1, \tau), \quad B u(\lambda, \tau)=e^{\pi i \kappa\left(\lambda+\frac{\tau}{2}\right)} u(\lambda+\tau, \tau), \\
T u(\lambda, \tau)=u(\lambda, \tau+1), \quad S u(\lambda, \tau)=e^{-\pi i \kappa \frac{\lambda^{2}}{2 \tau}} \tau^{-\frac{1}{2}-\frac{p(p+1)}{\kappa}} u\left(\frac{\lambda}{\tau},-\frac{1}{\tau}\right),
\end{gathered}
$$

where we fix $\arg \tau \in(0, \pi)$.

Proposition 4.1. If $u(\lambda, \tau)$ is a solution of the $K Z B$-heat equation, then $A u(\lambda, \tau)$, $B u(\lambda, \tau), T u(\lambda, \tau)$, and $S u(\lambda, \tau)$ are solutions too. Moreover, the transformations $A, B, T$ and $S$ preserve the properties (i)-(iv).

The proofs that $T$ and $S$ preserve the space of conformal blocks are given in [EK]. The proofs that $A$ and $B$ also preserve this space are straightforward and follow from the equations

$$
\vartheta_{1}(\lambda+1, \tau)=-\vartheta_{1}(\lambda, \tau), \quad \vartheta_{1}(\lambda+\tau, \tau)=-e^{-\pi i(2 \lambda+\tau)} \vartheta_{1}(\lambda, \tau) .
$$

Lemma 4.2. Restricted to the space of conformal blocks, the transformations $A, B, T$, and $S$ satisfy the relations

$$
\begin{gathered}
A^{2}=I, \quad B^{2}=I, \quad S^{2}=(-1)^{p} i e^{-\pi i \frac{p(p+1)}{\kappa}} I, \quad(S T)^{3}=(-1)^{p} i e^{-\pi i \frac{p(p+1)}{\kappa}} I, \\
T A=A T, \quad S A S^{-1}=B, \quad A B=(-1)^{\kappa} B A, \quad T B=i^{\kappa} B A T,
\end{gathered}
$$

where $I$ denotes the identity transformation.

Lemma 4.3. We have

$$
A u_{\kappa, n}(\lambda, \tau)=(-1)^{n} u_{\kappa, n}(\lambda, \tau), \quad B u_{\kappa, n}(\lambda, \tau)=-e^{2 \pi i \frac{p n}{\kappa}} u_{\kappa, \kappa-n}(\lambda, \tau) .
$$

Let $\left(t_{m, n}\right)$ and $\left(s_{m, n}\right)$ be the matrices of the transformations $T$ and $S$, respectively, with respect to the basis $\left\{u_{\kappa, n}(\lambda, \tau) \mid p+1 \leq n \leq \kappa-p-1\right\}$, namely, $T u_{\kappa, n}=\sum_{m=p+1}^{\kappa-p-1} t_{m, n} u_{\kappa, m}, S u_{\kappa, n}=\sum_{m=p+1}^{\kappa-p-1} s_{m, n} u_{\kappa, m}$. In Theorem 4.4, we give formulas for the matrices of $T$ and $S$ in terms of Macdonald polynomials of type $A_{1}$. 
The Macdonald polynomials [Ma] of type $A_{1}$ are $x$-even polynomials in terms of $\epsilon^{m x}$, where $m \in \mathbb{Z}$. They depend on two parameters $k$ and $n$, where $k$ and $n$ are non-negative integers. They are defined by the conditions:

1. $P_{n}^{(k)}(x)=\epsilon^{n x}+\epsilon^{-n x}+$ lower order terms, except for $P_{0}^{(k)}(x)=1$,

2. $\left\langle P_{m}^{(k)}, P_{n}^{(k)}\right\rangle=0$ for $m \neq n$, where

$$
\langle f, g\rangle=\frac{1}{2} \text { Const Term }\left(f g \prod_{j=0}^{k-1}\left(1-\epsilon^{2(j+x)}\right)\left(1-\epsilon^{2(j-x)}\right)\right) .
$$

Theorem 4.4. [FSV2] Let $\epsilon=e^{\pi i / \kappa}$. For $p+1 \leq m, n \leq \kappa-p-1$, we have

$$
\begin{gathered}
t_{m, n}=\epsilon^{\frac{n^{2}}{2}} \delta_{m n}, \\
s_{m, n}=\frac{e^{-\frac{\pi i}{4}}}{\sqrt{2 \kappa}} \epsilon^{p(n-m)-\frac{p(p+1)}{2}}\left(\epsilon^{-m}-\epsilon^{m}\right)\left(\prod_{j=1}^{p}\left(\epsilon^{-n+j}-\epsilon^{n-j}\right)\right) P_{n-p-1}^{(p+1)}(m),
\end{gathered}
$$

where $\delta_{m n}=1$ for $m=n$ and 0 otherwise.

\section{Integral identities}

To formulate our main result, we need functions $\eta, \phi_{1}, \phi_{2}$, and $\phi_{3}$. The Dedekind $\eta$-function is the function $\eta(\tau)=q^{1 / 24} \prod_{j=1}^{\infty}\left(1-q^{j}\right)$. We have

$$
\vartheta_{1}^{\prime}(0, \tau)=2 \pi \eta^{3}(\tau)
$$

Consider the functions $[\mathrm{W}]$

$$
\begin{aligned}
& \phi_{1}(\tau)=\frac{\eta(\tau)^{2}}{\eta\left(\frac{\tau}{2}\right) \eta(2 \tau)}=q^{-\frac{1}{48}} \prod_{j=1}^{\infty}\left(1+q^{j-\frac{1}{2}}\right), \\
& \phi_{2}(\tau)=\frac{\eta\left(\frac{\tau}{2}\right)}{\eta(\tau)}=q^{-\frac{1}{48}} \prod_{j=1}^{\infty}\left(1-q^{j-\frac{1}{2}}\right), \\
& \phi_{3}(\tau)=\sqrt{2} \frac{\eta(2 \tau)}{\eta(\tau)}=\sqrt{2} q^{\frac{1}{24}} \prod_{j=1}^{\infty}\left(1+q^{j}\right) .
\end{aligned}
$$

We have

$$
\begin{gathered}
\phi_{1}\left(-\frac{1}{\tau}\right)=\phi_{1}(\tau), \quad \phi_{2}\left(-\frac{1}{\tau}\right)=\phi_{3}(\tau), \quad \phi_{3}\left(-\frac{1}{\tau}\right)=\phi_{2}(\tau), \\
\phi_{1}(\tau+1)=e^{-\frac{\pi i}{24}} \phi_{2}(\tau), \quad \phi_{2}(\tau+1)=e^{-\frac{\pi i}{24}} \phi_{1}(\tau), \quad \phi_{3}(\tau+1)=e^{\frac{\pi i}{12}} \phi_{3}(\tau) .
\end{gathered}
$$

Let

$$
\begin{aligned}
& c_{\kappa, n}= \\
& c_{p, \kappa, n}=(2 \pi)^{\frac{p(p+1)}{\kappa}} e^{-\pi i \frac{p(3 p-1)}{2 \kappa}} e^{\pi i \frac{p+1}{2}} B_{p}\left(\frac{n+1}{\kappa},-\frac{2 p}{\kappa}, \frac{1}{\kappa}\right) \prod_{j=1}^{p}\left(1-e^{2 \pi i \frac{n+j}{\kappa}}\right) .
\end{aligned}
$$

Here, $B_{p}(\alpha, \beta, \gamma)$ is the Selberg integral. 
Theorem 5.1. We have ten series of identities,

$$
u_{2 p+4, p+2}(\lambda, \tau)=2^{-\frac{2(p+1)}{2 p+4}} c_{2 p+4, p+2}\left(\phi_{3}(\tau) \eta(\tau)^{-1}\right)^{\frac{4(p+1)}{2 p+4}} \vartheta_{1}^{p+1}(\lambda, \tau) \theta_{2,1}^{s}(\lambda, \tau)
$$

$$
\begin{gathered}
u_{2 p+4, p+1}(\lambda, \tau)+(-1)^{p+1} e^{2 \pi i \frac{p(p+1)}{2 p+4}} u_{2 p+4, p+3}(\lambda, \tau)= \\
c_{2 p+4, p+1}\left(\phi_{2}(\tau) \eta(\tau)^{-1}\right)^{\frac{4(p+1)}{2 p+4}} \vartheta_{1}^{p+1}(\lambda, \tau)\left(\theta_{2,0}(\lambda, \tau)-\theta_{2,2}(\lambda, \tau)\right) \\
u_{2 p+4, p+1}(\lambda, \tau)+(-1)^{p} e^{2 \pi i \frac{p(p+1)}{2 p+4}} u_{2 p+4, p+3}(\lambda, \tau)= \\
c_{2 p+4, p+1}\left(\phi_{1}(\tau) \eta(\tau)^{-1}\right)^{\frac{4(p+1)}{2 p+4}} \vartheta_{1}^{p+1}(\lambda, \tau)\left(\theta_{2,0}(\lambda, \tau)+\theta_{2,2}(\lambda, \tau)\right)
\end{gathered}
$$

$$
\begin{aligned}
& u_{2 p+6, p+1}(\lambda, \tau)+(-1)^{p+1} e^{2 \pi i \frac{p(p+1)}{2 p+6}} u_{2 p+6, p+5}(\lambda, \tau)= \\
& 2^{\frac{3(p+1)}{2 p+6}} c_{2 p+6, p+1}\left(\phi_{3}(\tau) \eta(\tau)\right)^{-\frac{6(p+1)}{2 p+6}} \vartheta_{1}^{p+1}(\lambda, \tau)\left(\theta_{4,0}(\lambda, \tau)-\theta_{4,4}(\lambda, \tau)\right),
\end{aligned}
$$

$$
\begin{aligned}
& u_{2 p+6, p+2}(\lambda, \tau)+(-1)^{p} e^{2 \pi i \frac{p(p+2)}{2 p+6}} u_{2 p+6, p+4}(\lambda, \tau)= \\
& c_{2 p+6, p+2}\left(\phi_{2}(\tau) \eta(\tau)\right)^{-\frac{6(p+1)}{2 p+6}} \vartheta_{1}^{p+1}(\lambda, \tau)\left(\theta_{4,1}^{s}(\lambda, \tau)+\theta_{4,3}^{s}(\lambda, \tau)\right)
\end{aligned}
$$

$$
\begin{aligned}
& u_{2 p+6, p+2}(\lambda, \tau)+(-1)^{p+1} e^{2 \pi i \frac{p(p+2)}{2 p+6}} u_{2 p+6, p+4}(\lambda, \tau)= \\
& c_{2 p+6, p+2}\left(\phi_{1}(\tau) \eta(\tau)\right)^{-\frac{6(p+1)}{2 p+6}} \vartheta_{1}^{p+1}(\lambda, \tau)\left(\theta_{4,1}^{s}(\lambda, \tau)-\theta_{4,3}^{s}(\lambda, \tau)\right),
\end{aligned}
$$

$$
\begin{aligned}
u_{2 p+8, p+2}(\lambda, \tau)+ & (-1)^{p+1} e^{2 \pi i \frac{p(p+2)}{2 p+8}} u_{2 p+8, p+6}(\lambda, \tau)= \\
& c_{2 p+8, p+2} \eta(\tau)^{\frac{-8(p+1)}{2 p+8}} \vartheta_{1}^{p+1}(\lambda, \tau)\left(\theta_{6,1}^{s}(\lambda, \tau)-\theta_{6,5}^{s}(\lambda, \tau)\right) .
\end{aligned}
$$

The integrals in Theorem 5.1 are appropriately called the elliptic Selberg integrals. The identity (2) appears in [FSV1] and in [FV1] for $p=1$.

\section{Differential equations}

In Lemmas 6.1-6.3, let ' denote the derivative with respect to $\lambda$, let ' denote the derivative with respect to $\tau$, and let

$$
v(\lambda, \tau)=\vartheta_{1}^{p+1}(\lambda, \tau) \sum_{j=0}^{\kappa-2 p-2} c_{j}(\tau) \theta_{\kappa-2 p-2, j}^{s}(\lambda, \tau) .
$$


Lemma 6.1. The function $v(\lambda, \tau)$ is a solution of the KZB-heat equation if and only if the differential equation

$$
\begin{aligned}
\frac{\kappa}{p+1} \sum_{j=0}^{\kappa-2 p-2}\left(\frac{d}{d \tau} c_{j}\right) \theta_{\kappa-2 p-2, j}^{s}=(2 p+2-\kappa) \frac{\dot{\vartheta}_{1}}{\vartheta_{1}} \sum_{j=0}^{\kappa-2 p-2} c_{j} \theta_{\kappa-2 p-2, j}^{s} \\
-2 \sum_{j=0}^{\kappa-2 p-2} c_{j} \dot{\theta}_{\kappa-2 p-2, j}^{s}+\frac{1}{\pi i} \frac{\vartheta_{1}^{\prime}}{\vartheta_{1}} \sum_{j=0}^{\kappa-2 p-2} c_{j}\left(\theta_{\kappa-2 p-2, j}^{s}\right)^{\prime}
\end{aligned}
$$

holds.

The proof of Lemma 6.1 uses the identities

$$
\begin{gathered}
2 \pi i(2 p+2)\left(\vartheta_{1}^{\dot{p+1}}\right)(\lambda, \tau)=\left(\vartheta_{1}^{p+1}\right)^{\prime \prime}(\lambda, \tau)+p(p+1) \rho^{\prime}(\lambda, \tau) \vartheta_{1}^{p+1}(\lambda, \tau), \\
2 \pi i \kappa \dot{\theta}_{\kappa, m}^{s}(\lambda, \tau)=\left(\theta_{\kappa, m}^{s}\right)^{\prime \prime}(\lambda, \tau) .
\end{gathered}
$$

Proof of Lemma 6.1. Applying the differential operator $2 \pi i \kappa \partial / \partial \tau$ to $v(\lambda, \tau)$ gives

$$
\begin{aligned}
2 \pi i \kappa\left[(p+1) \dot{\vartheta}_{1} \vartheta_{1}^{p}\right. & \sum_{j=0}^{\kappa-2 p-2} c_{j} \theta_{\kappa-2 p-2, j}^{s}+\vartheta_{1}^{p+1} \sum_{j=0}^{\kappa-2 p-2}\left(\frac{d}{d \tau} c_{j}\right) \theta_{\kappa-2 p-2, j}^{s} \\
& \left.+\vartheta_{1}^{p+1} \sum_{j=0}^{\kappa-2 p-2} c_{j} \dot{\theta}_{\kappa-2 p-2, j}^{s}\right] .
\end{aligned}
$$

Applying the differential operator $\partial^{2} / \partial \lambda^{2}+p(p+1) \rho^{\prime}(\lambda, \tau)$ to $v(\lambda, \tau)$ gives

$$
\begin{aligned}
\left(\vartheta_{1}^{p+1}\right)^{\prime \prime} & \sum_{j=0}^{\kappa-2 p-2} c_{j} \theta_{\kappa-2 p-2, j}^{s}+2(p+1) \vartheta_{1}^{\prime} \vartheta_{1}^{p} \sum_{j=0}^{\kappa-2 p-2} c_{j}\left(\theta_{\kappa-2 p-2, j}^{s}\right)^{\prime} \\
& +\vartheta_{1}^{p+1} \sum_{j=0}^{\kappa-2 p-2} c_{j}\left(\theta_{\kappa-2 p-2, j}^{s}\right)^{\prime \prime}+p(p+1) \rho^{\prime} \vartheta_{1}^{p+1} \sum_{j=0}^{\kappa-2 p-2} c_{j} \theta_{\kappa-2 p-2, j}^{s} .
\end{aligned}
$$

Applying (13) and (14), we obtain the result.

Lemma 6.2. If $v(\lambda, \tau)$ is a solution of the KZB-heat equation, then the functions $c_{j}(\tau)$ satisfy the differential equation

$$
\begin{aligned}
\frac{\kappa}{p+1} \sum_{j=0}^{\kappa-2 p-2}\left(\frac{d}{d \tau} c_{j}(\tau)\right) \theta_{\kappa-2 p-2, j}(0, \tau) & \\
(2 p+2-\kappa)\left(\frac{d}{d \tau} \ln \vartheta_{1}^{\prime}(0, \tau)\right) & \sum_{j=0}^{\kappa-2 p-2} c_{j}(\tau) \theta_{\kappa-2 p-2, j}(0, \tau) \\
& +2(\kappa-2 p-3) \sum_{j=0}^{\kappa-2 p-2} c_{j}(\tau) \frac{d}{d \tau} \theta_{\kappa-2 p-2, j}(0, \tau) .
\end{aligned}
$$


Proof. For any fixed $\lambda$, equation (12) gives a differential equation for the functions $c_{j}(\tau)$. We take the limit of that equation as $\lambda \rightarrow 0$. In the ratio $\dot{\vartheta}_{1} / \vartheta_{1}$, both the numerator and denominator tend to zero, so the limit of this term as $\lambda \rightarrow 0$ is equal to the limit of the ratio of the derivatives of the numerator and the denominator. The limit of the ratio $\left(\sum_{j=0}^{\kappa-2 p-2} c_{j}\left(\theta_{\kappa-2 p-2, j}^{s}\right)^{\prime}\right) / \vartheta_{1}$ is calculated in the same way, since each $\theta_{\kappa-2 p-2, j}^{s}$ is a symmetric function and therefore $\left(\theta_{\kappa-2 p-2, j}^{s}\right)^{\prime}(0, \tau)=0$. Then the result follows from (14).

Lemma 6.3. If $v(\lambda, \tau)$ is a solution of the KZB-heat equation, then the functions $c_{j}(\tau)$ satisfy the differential equation

$$
\begin{aligned}
\frac{\kappa}{p+1} \sum_{j=0}^{\kappa-2 p-2}\left(\frac{d}{d \tau} c_{j}(\tau)\right) \theta_{\kappa-2 p-2, \kappa-2 p-2-j}(0, \tau)= \\
(2 p+2-\kappa)\left(\frac{d}{d \tau} \ln \vartheta_{1}^{\prime}(0, \tau)\right) \sum_{j=0}^{\kappa-2 p-2} c_{j}(\tau) \theta_{\kappa-2 p-2, \kappa-2 p-2-j}(0, \tau) \\
+2(\kappa-2 p-3) \sum_{j=0}^{\kappa-2 p-2} c_{j}(\tau) \frac{d}{d \tau} \theta_{\kappa-2 p-2, \kappa-2 p-2-j}(0, \tau) .
\end{aligned}
$$

Proof. We take the limit of (12) as $\lambda \rightarrow \tau$. This limit is calculated in terms of the limit $\lambda \rightarrow 0$ using the formulas

$$
\begin{gathered}
\left.\frac{\partial}{\partial z} \theta_{1}(z, \tau)\right|_{z=\lambda+\tau}=e^{-2 \pi i \lambda-\pi i \tau}\left(2 \pi i \theta_{1}(\lambda, \tau)-\theta_{1}^{\prime}(\lambda, \tau)\right), \\
\left.\frac{\partial}{\partial z} \theta_{1}(\lambda+\tau, z)\right|_{z=\tau}=e^{-2 \pi i \lambda-\pi i \tau}\left(-\pi i \theta_{1}(\lambda, \tau)+\theta_{1}^{\prime}(\lambda, \tau)-\dot{\theta}_{1}(\lambda, \tau)\right), \\
\left.\frac{\partial}{\partial z} \theta_{\kappa, m}^{s}(z, \tau)\right|_{z=\lambda+\tau}=e^{-\pi i \kappa \lambda-\pi i \kappa \frac{\tau}{2}}\left(-\pi i \kappa \theta_{\kappa, \kappa-m}^{s}(\lambda, \tau)+\left(\theta_{\kappa, \kappa-m}^{s}\right)^{\prime}(\lambda, \tau)\right), \\
\left.\frac{\partial}{\partial z} \theta_{\kappa, m}^{s}(\lambda+\tau, z)\right|_{z=\tau}= \\
e^{-\pi i \kappa \lambda-\pi i \kappa \frac{\tau}{2}}\left(\pi i \frac{\kappa}{2} \theta_{\kappa, \kappa-m}^{s}(\lambda, \tau)-\left(\theta_{\kappa, \kappa-m}^{s}\right)^{\prime}(\lambda, \tau)+\dot{\theta}_{\kappa, \kappa-m}^{s}(\lambda, \tau)\right) .
\end{gathered}
$$

It is straightforward to calculate the limit of the left hand side. Using the above formulas, the limit as $\lambda \rightarrow \tau$ of the right hand side is equal to the limit as $\lambda \rightarrow 0$ of the expression

$$
\begin{aligned}
& e^{\pi i(2 p+2-\kappa)\left(\lambda+\frac{\tau}{2}\right)}\left((2 p+2-\kappa) \frac{\dot{\vartheta}_{1}}{\vartheta_{1}} \sum_{j=0}^{\kappa-2 p-2} c_{j} \theta_{\kappa-2 p-2, \kappa-2 p-2-j}^{s}\right. \\
& \left.-2 \sum_{j=0}^{\kappa-2 p-2} c_{j} \dot{\theta}_{\kappa-2 p-2, \kappa-2 p-2-j}^{s}+\frac{1}{\pi i} \frac{\vartheta_{1}^{\prime}}{\vartheta_{1}} \sum_{j=0}^{\kappa-2 p-2} c_{j}\left(\theta_{\kappa-2 p-2, \kappa-2 p-2-j}^{s}\right)^{\prime}\right) .
\end{aligned}
$$

This limit is calculated using L'Hôpital's rule. 


\section{Identities for theta functions}

In the next section, we give the proofs of the integral identities in Theorem 5.1. We will use the following results.

Lemma 7.1. We have $\theta_{2,1}^{s}(\lambda)=\vartheta_{1}(\lambda+1 / 2)$.

Lemma 7.1 is proved by comparing the Fourier series expansions of the functions.

Corollary 7.2. We have $2 \theta_{2,1}(0)=\eta(\tau) \phi_{3}(\tau)^{2}$.

Lemma 7.3. Let

$f_{1}(\tau)=\frac{\theta_{4,1}(0)-\theta_{4,3}(0)}{\eta(\tau)}, \quad f_{2}(\tau)=\frac{\theta_{4,1}(0)+\theta_{4,3}(0)}{\eta(\tau)}, \quad f_{3}(\tau)=\frac{\theta_{4,0}(0)-\theta_{4,4}(0)}{\sqrt{2} \eta(\tau)}$.

Then $f_{1}(\tau)=\phi_{1}(\tau)^{-1}, f_{2}(\tau)=\phi_{2}(\tau)^{-1}, f_{3}(\tau)=\phi_{3}(\tau)^{-1}$.

The proof of Lemma 7.3 is based on the following result.

Lemma 7.4. [W] Suppose $g_{1}(\tau), g_{2}(\tau)$, and $g_{3}(\tau)$ are holomorphic functions on the upper half plane $\mathbb{C}_{+}$satisfying the following conditions.

$\mathrm{P} 1$. The functions $g_{1}, g_{2}$, and $g_{3}$ can be written in the forms

$$
g_{1}(\tau)=q^{-\frac{a}{48}} \sum_{j=0}^{\infty} a_{j} q^{\frac{j}{2}}, \quad g_{2}(\tau)=q^{-\frac{a}{48}} \sum_{j=0}^{\infty}(-1)^{j} a_{j} q^{\frac{j}{2}}, \quad g_{3}(\tau)=q^{\frac{a}{24}} \sum_{j=0}^{\infty} b_{j} q^{j},
$$

where $a$ is an integer, $a_{j}, b_{j} \in \mathbb{C}$, and $a_{0}=1$.

$\mathrm{P} 2$. The functions $g_{1}, g_{2}$, and $g_{3}$ have the modular properties

$$
g_{1}\left(-\frac{1}{\tau}\right)=g_{1}(\tau), \quad g_{2}\left(-\frac{1}{\tau}\right)=g_{3}(\tau), \quad g_{3}\left(-\frac{1}{\tau}\right)=g_{2}(\tau) .
$$

Then $g_{i}(\tau)=\phi_{i}(\tau)^{a}$, for $i=1,2,3$.

Proof of Lemma 7.3. We have

$$
\begin{gathered}
\theta_{4,1}(0)=\sum_{j \in \mathbb{Z}} q^{4\left(j+\frac{1}{8}\right)^{2}}=q^{\frac{1}{16}} \sum_{j \in \mathbb{Z}} q^{\frac{1}{2}\left(8 j^{2}+2 j\right)}, \\
\theta_{4,3}(0)=\sum_{j \in \mathbb{Z}} q^{4\left(j+\frac{3}{8}\right)^{2}}=q^{\frac{1}{16}} \sum_{j \in \mathbb{Z}} q^{\frac{1}{2}\left(8 j^{2}+6 j+1\right)}, \\
\theta_{4,0}(0)=\sum_{j \in \mathbb{Z}} q^{4 j^{2}}=\sum_{j \in \mathbb{Z}} q^{(2 j)^{2}}, \\
\theta_{4,4}(0)=\sum_{j \in \mathbb{Z}} q^{4\left(j+\frac{1}{2}\right)^{2}}=\sum_{j \in \mathbb{Z}} q^{(2 j+1)^{2}} .
\end{gathered}
$$

Hence, the functions

$$
\begin{gathered}
\tilde{f}_{1}(\tau)=q^{-\frac{1}{24}}\left(\theta_{4,1}(0)-\theta_{4,3}(0)\right), \\
\tilde{f}_{2}(\tau)=q^{-\frac{1}{24}}\left(\theta_{4,1}(0)+\theta_{4,3}(0)\right), \\
\tilde{f}_{3}(\tau)=2^{-\frac{1}{2}} q^{-\frac{1}{24}}\left(\theta_{4,0}(0)-\theta_{4,4}(0)\right)
\end{gathered}
$$


are holomorphic functions on $\mathbb{C}_{+}$with the property P1 for $a=-1$ and $g_{i}=\tilde{f}_{i}$. Let $y(q)=\sum_{j=1}^{\infty} c_{j} q^{j}$ be defined by the condition $1+y(q)=q^{-1 / 24} \eta(\tau)$. Then for $i=1,2,3$,

$$
f_{i}(\tau)=\frac{\tilde{f}_{i}(\tau)}{1+y(q)}=\tilde{f}_{i}(\tau)\left(1-y(q)+y(q)^{2}+\ldots\right)
$$

are holomorphic functions on $\mathbb{C}_{+}$with the property $\mathrm{P} 1$ for $a=-1$ and $g_{i}=f_{i}$. One checks that $f_{1}, f_{2}$ and $f_{3}$ have the property $\mathrm{P} 2$ using the modular properties of $\theta_{4, n}(0)$ and $\eta(\tau)$.

Lemma 7.5. We have $\theta_{6,1}(0)-\theta_{6,5}(0)=\eta(\tau)$.

Lemma 7.5 is proved by comparing the infinite series expansions of the functions.

\section{The proof of Theorem 5.1}

Proof of (2). For $\kappa=2 p+2$, the space of conformal blocks is one-dimensional. The right hand side of (2) is a solution of (1) with the properties (i)-(iv) [FV1]. According to Theorem 3.1, the left hand side also has these properties. Thus the two functions are proportional. The coefficient of proportionality is calculated by comparing the leading terms of $\vartheta_{1}^{p+1}$ and $u_{\kappa, p+1}$ in the limit as $\tau \rightarrow i \infty$. The leading term of $\vartheta_{1}^{p+1}$ is $(-i)^{p+1} q^{(p+1) / 8}\left(e^{\pi i \lambda}-e^{-\pi i \lambda}\right)^{p+1}$. Let $d t=d t_{1} \ldots d t_{p}$. The leading term of $u_{\kappa, p+1}$ is

$$
\begin{gathered}
\int_{\Delta_{p}} \prod_{j=1}^{p}\left(\frac{e^{\pi i t_{j}}-e^{-\pi i t_{j}}}{2 \pi e^{\frac{\pi i}{2}}}\right)^{-\frac{2 p}{2 p+2}-1} \prod_{1 \leq j<k \leq p}\left(\frac{e^{\pi i\left(t_{j}-t_{k}\right)}-e^{-\pi i\left(t_{j}-t_{k}\right)}}{2 \pi e^{\frac{\pi i}{2}}}\right)^{\frac{2}{2 p+2}} \\
\left(\left(\prod_{j=1}^{p} \frac{e^{\pi i\left(\lambda-t_{j}\right)}-e^{-\pi i\left(\lambda-t_{j}\right)}}{e^{\pi i \lambda}-e^{-\pi i \lambda}}\right) q^{\frac{(p+1)^{2}}{4(2 p+2)}} e^{\pi i(p+1)\left(\lambda+\frac{2}{2 p+2} \sum_{j=1}^{p} t_{j}\right)}\right. \\
\left.+(-1)^{p+1}\left(\prod_{j=1}^{p} \frac{e^{\pi i\left(\lambda+t_{j}\right)}-e^{-\pi i\left(\lambda+t_{j}\right)}}{e^{\pi i \lambda}-e^{-\pi i \lambda}}\right) q^{\frac{(p+1)^{2}}{4(2 p+2)}} e^{\pi i(p+1)\left(-\lambda+\frac{2}{2 p+2} \sum_{j=1}^{p} t_{j}\right)}\right) d t .
\end{gathered}
$$

The above expression is equal to

$$
\left(2 \pi e^{\frac{\pi i}{2}}\right)^{\frac{p(p+1)}{2 p+2}+p} e^{-\pi i\left(\frac{2 p^{2}}{2 p+2}+p\right)} q^{\frac{p+1}{8}}\left(e^{\pi i \lambda}-e^{-\pi i \lambda}\right)^{-p} \sum_{l=0}^{p} I_{l}\left(e^{\pi i(2 l+1) \lambda}-e^{-\pi i(2 l+1) \lambda}\right),
$$

where

$$
\begin{gathered}
I_{l}=\int_{\Delta_{p}} f_{l}\left(t_{1}, \ldots, t_{p}\right) \prod_{j=1}^{p} e^{2 \pi i t_{j}\left(\frac{p+2}{2 p+2}+\frac{1}{2}\right)}\left(1-e^{2 \pi i t_{j}}\right)^{-\frac{2 p}{2 p+2}-1} \times \\
\prod_{1 \leq j<k \leq p}\left(e^{2 \pi i t_{j}}-e^{2 \pi i t_{k}}\right)^{\frac{2}{2 p+2}} d t
\end{gathered}
$$


for some function $f_{l}$, symmetric in the variables $t_{1}, \ldots, t_{p}$. Comparing the coefficients of $e^{\pi i(p+1) \lambda}$ in the leading terms, we find that

$$
u_{\kappa, p+1}=i^{p+1}\left(2 \pi e^{\frac{\pi i}{2}}\right)^{\frac{p(p+1)}{2 p+2}+p} e^{-\pi i\left(\frac{2 p^{2}}{2 p+2}+p\right)} I_{p} \vartheta_{1}^{p+1} .
$$

To complete the proof, it remains to compute $I_{p}$. It is not difficult to show that $f_{p}\left(t_{1}, \ldots, t_{p}\right)=\prod_{j=1}^{p} e^{-\pi i t_{j}}$. Let $x_{j}=e^{2 \pi i t_{j}}$. Let $\tilde{\Delta}_{p}$ be the image of $\Delta_{p}$ under the map $t_{j} \mapsto x_{j}$. We have

$$
I_{p}=(2 \pi i)^{-p} \int_{\tilde{\Delta}_{p}} \prod_{j=1}^{p} x_{j}^{\frac{p+2}{2 p+2}-1}\left(1-x_{j}\right)^{-\frac{2 p}{2 p+2}-1} \prod_{1 \leq j<k \leq p}\left(x_{j}-x_{k}\right)^{\frac{2}{2 p+2}} d x .
$$

Applying the Stokes theorem, we deform the contour $\tilde{\Delta}_{p}$ to get

$$
\begin{aligned}
I_{p}=(2 \pi i)^{-p} & \prod_{j=1}^{p}\left(e^{2 \pi i \frac{j+p+1}{2 p+2}}-1\right) \times \\
& \int_{\Delta_{p}} \prod_{j=1}^{p} x_{j}^{\frac{p+2}{2 p+2}-1}\left(1-x_{j}\right)^{-\frac{2 p}{2 p+2}-1} \prod_{1 \leq j<k \leq p}\left(x_{j}-x_{k}\right)^{\frac{2}{2 p+2}} d x .
\end{aligned}
$$

Observe that

$$
\int_{\Delta_{p}} \prod_{j=1}^{p} x_{j}^{\frac{p+2}{2 p+2}-1}\left(1-x_{j}\right)^{-\frac{2 p}{2 p+2}-1} \prod_{1 \leq j<k \leq p}\left(x_{j}-x_{k}\right)^{\frac{2}{2 p+2}} d x
$$

is the Selberg integral $B_{p}((p+2) /(2 p+2),-2 p /(2 p+2), 1 /(2 p+2))$. This completes the proof.

Proof of (3) and (4). Let $\kappa=2 p+3$. Then any solution of (1) with the properties (i)-(iv) has the form

$$
v(\lambda, \tau)=\vartheta_{1}^{p+1}(\lambda, \tau)\left(c_{0}(\tau) \theta_{1,0}(\lambda, \tau)+c_{1}(\tau) \theta_{1,1}(\lambda, \tau)\right) .
$$

Let $A$ be the transformation introduced in section 4. By Proposition 4.1, $A v(\lambda, \tau)=(-1)^{p+1} \vartheta_{1}^{p+1}(\lambda, \tau)\left(c_{0}(\tau) \theta_{1,0}(\lambda, \tau)-c_{1}(\tau) \theta_{1,1}(\lambda, \tau)\right)$ is also a solution. Hence, for $j=0$ or 1 , the function $v_{j}(\lambda, \tau)=c_{j}(\tau) \vartheta_{1}^{p+1}(\lambda, \tau) \theta_{1, j}(\lambda, \tau)$ gives a solution too. Moreover, $A v_{j}=(-1)^{p+1+j} v_{j}$. By Theorem 3.2, the integrals $u_{\kappa, p+1}$ and $u_{\kappa, p+2}$ span the space of conformal blocks. By Lemma 4.3, $A u_{\kappa, p+1}=(-1)^{p+1} u_{\kappa, p+1}$ and $A u_{\kappa, p+2}=(-1)^{p} u_{\kappa, p+2}$. So for $j=0$ or 1 , the integral $u_{\kappa, p+1+j}$ is proportional to $v_{j}$. By Lemma $6.2, c_{j}(\tau)$ must satisfy the differential equation

$$
\frac{\kappa}{p+1}\left(\frac{d}{d \tau} c_{j}(\tau)\right) \theta_{1, j}(0, \tau)=-\left(\frac{d}{d \tau} \ln \vartheta_{1}^{\prime}(0, \tau)\right) c_{j}(\tau) \theta_{1, j}(0, \tau) .
$$

The function $c_{j}(\tau)=\left((2 \pi)^{-1} \vartheta_{1}^{\prime}(0, \tau)\right)^{-(p+1) / \kappa}=\eta(\tau)^{-3(p+1) / \kappa}$ is a solution of this equation for $j=0$ and $j=1$. The coefficients of proportionality are computed in the limit as $\tau \rightarrow i \infty$, cf. the proof of (2). This completes the proof. 
Proof of (5), (6), and (7). Let $\kappa=2 p+4$. Let

$$
v_{j}(\lambda, \tau)=\vartheta_{1}^{p+1}(\lambda, \tau) \theta_{2, j}^{s}(\lambda, \tau), 0 \leq j \leq 2 .
$$

Any solution of (1) with the properties (i)-(iv) has the form $v(\lambda, \tau)=$ $\sum_{j=0}^{2} c_{j}(\tau) v_{j}(\lambda, \tau)$. Let $A$ be the transformation in section 4. By Proposition 4.1, $A v=\sum_{j=0}^{2}(-1)^{p+j+1} c_{j} v_{j}$ is also a solution. Hence the function $c_{1} v_{1}$ gives a solution too. Moreover, it is an eigenvector of $A$ with eigenvalue $(-1)^{p}$. By Theorem 3.2, the space of conformal blocks is three-dimensional with spanning set $\left\{u_{\kappa, n} \mid p+1 \leq n \leq p+3\right\}$. According to Lemma 4.3, the eigenspace of $A$ corresponding to the eigenvalue $(-1)^{p}$ is one-dimensional and is spanned by $u_{\kappa, p+2}$. It follows that $u_{\kappa, p+2}$ is proportional to $c_{1} v_{1}$. By Lemma $6.2, c_{1}(\tau)$ must satisfy the differential equation

$$
\begin{aligned}
& \frac{\kappa}{p+1}\left(\frac{d}{d \tau} c_{1}(\tau)\right) \theta_{2,1}(0, \tau)= \\
&-2\left(\frac{d}{d \tau} \ln \vartheta_{1}^{\prime}(0, \tau)\right) c_{1}(\tau) \theta_{2,1}(0, \tau)+2 c_{1}(\tau) \frac{d}{d \tau} \theta_{2,1}(0, \tau)
\end{aligned}
$$

The function $c_{1}(\tau)=\left(4 \pi \theta_{2,1}(0, \tau) \vartheta_{1}^{\prime}(0, \tau)^{-1}\right)^{2(p+1) / \kappa}$ is a solution of the above equation. By Corollary 7.2, $c_{1}(\tau)=\left(\phi_{3}(\tau) \eta(\tau)^{-1}\right)^{4(p+1) / \kappa}$. The coefficient of proportionality is computed in the limit $\tau \rightarrow i \infty$, cf. the proof of (2). This proves (5). To prove (6), we apply the transformation $S$ to both sides of (5). To prove (7), we apply the transformation $T$ to both sides of (6). This completes the proof.

Proof of (8), (9), and (10). Let $\kappa=2 p+6$. Let

$$
v_{j}(\lambda, \tau)=\vartheta_{1}^{p+1}(\lambda, \tau) \theta_{4, j}^{s}(\lambda, \tau), 0 \leq j \leq 4
$$

Any solution of (1) with the properties (i)-(iv) has the form $v(\lambda, \tau)=$ $\sum_{j=0}^{4} c_{j}(\tau) v_{j}(\lambda, \tau)$. Let $A$ and $B$ be the transformations in section 4 . By Proposition 4.1, $A v=\sum_{j=0}^{4}(-1)^{p+j+1} c_{j} v_{j}$ is also a solution. Hence the function $c_{0} v_{0}+c_{2} v_{2}+c_{4} v_{4}$ gives a solution too. Moreover, $B\left(c_{0} v_{0}+c_{2} v_{2}+c_{4} v_{4}\right)=$ $(-1)^{p+1}\left(c_{4} v_{0}+c_{2} v_{2}+c_{0} v_{4}\right)$ is also a solution. So there exists a solution of the form $c(\tau)\left(v_{0}-v_{4}\right)$. It is an eigenvector of $A$ with eigenvalue $(-1)^{p+1}$ and an eigenvector of $\mathrm{B}$ with eigenvalue $(-1)^{p}$. We show that the subspace of conformal blocks with this property is one-dimensional. By Theorem 3.2, the space of conformal blocks is five-dimensional with spanning set $\left\{u_{\kappa, n} \mid p+1 \leq n \leq p+5\right\}$. By Lemma 4.3, the eigenspace of $A$ corresponding to the eigenvalue $(-1)^{p+1}$ is three-dimensional and is spanned by $u_{\kappa, p+1}, u_{\kappa, p+3}$, and $u_{\kappa, p+5}$. By Lemma 4.3 , the transformation $B$ preserves the subspace $\left\langle u_{\kappa, p+1}, u_{\kappa, p+3}, u_{\kappa, p+5}\right\rangle$. The 
matrix of $B$ restricted to this subspace is

$$
\left(\begin{array}{ccc}
0 & 0 & -e^{2 \pi i \frac{p(p+5)}{\kappa}} \\
0 & (-1)^{p+1} & 0 \\
-e^{2 \pi i \frac{p(p+1)}{\kappa}} & 0 & 0
\end{array}\right) .
$$

Thus the restriction of $B$ to $\left\langle u_{\kappa, p+1}, u_{\kappa, p+3}, u_{\kappa, p+5}\right\rangle$ has eigenvalues $(-1)^{p}$ and $(-1)^{p+1}$ of multiplicities 1 and 2 , respectively. The eigenspace corresponding to the eigenvalue $(-1)^{p}$ is spanned by the vector $u_{\kappa, p+1}+(-1)^{p+1} e^{2 \pi i p(p+1) / \kappa} u_{\kappa, p+5}$. It follows that this vector is proportional to $c(\tau)\left(v_{0}-v_{4}\right)$. By Lemma 6.2, $c(\tau)$ must satisfy the differential equation

$$
\begin{gathered}
\frac{\kappa}{p+1}\left(\frac{d}{d \tau} c(\tau)\right)\left(\theta_{4,0}(0, \tau)-\theta_{4,4}(0, \tau)\right)= \\
-4\left(\frac{d}{d \tau} \ln \vartheta_{1}^{\prime}(0, \tau)\right) c(\tau)\left(\theta_{4,0}(0, \tau)-\theta_{4,4}(0, \tau)\right)+6 c(\tau) \frac{d}{d \tau}\left(\theta_{4,0}(0, \tau)-\theta_{4,4}(0, \tau)\right) .
\end{gathered}
$$

The function $c(\tau)=\left((2 \pi)^{2}\left(\theta_{4,0}(0, \tau)-\theta_{4,4}(0, \tau)\right)^{3} \vartheta_{1}^{\prime}(0, \tau)^{-2}\right)^{2(p+1) / \kappa}$ is a solution of the above equation. By Lemma 7.3, we have

$$
c(\tau)=2^{3(p+1) / \kappa}\left(\phi_{3}(\tau) \eta(\tau)\right)^{-6(p+1) / \kappa} .
$$

The coefficient of proportionality is computed as in the proof of (2). This proves (8). To prove (9) and (10), apply the transformations $S$ and $T S$, respectively, to both sides of (8). This completes the proof.

Proof of (11). Let $\kappa=2 p+8$. Let $v_{j}(\lambda, \tau)=\vartheta_{1}^{p+1}(\lambda, \tau) \theta_{6, j}^{s}(\lambda, \tau), 0 \leq j \leq 6$. Any solution of (1) with the properties (i)-(iv) has the form

$$
v(\lambda, \tau)=\sum_{j=0}^{6} c_{j}(\tau) v_{j}(\lambda, \tau) .
$$

Let $A$ and $B$ be as in section 4. By Proposition 4.1, $A v=\sum_{j=0}^{6}(-1)^{p+j+1} c_{j} v_{j}$ is also a solution. Hence the function $c_{1} v_{1}+c_{3} v_{3}+c_{5} v_{5}$ gives a solution too. The function $B\left(c_{1} v_{1}+c_{3} v_{3}+c_{5} v_{5}\right)=(-1)^{p+1}\left(c_{5} v_{1}+c_{3} v_{3}+c_{1} v_{5}\right)$ also gives a solution. So there is a solution of the form $c(\tau)\left(v_{1}-v_{5}\right)$ which is an eigenvector of $A$ and $B$ with eigenvalue $(-1)^{p}$ under both transformations. We show that the subspace of conformal blocks with this property is one-dimensional. By Theorem 3.2, the space of conformal blocks is seven-dimensional with spanning set $\left\{u_{\kappa, n} \mid p+1 \leq n \leq p+7\right\}$. By Lemma 4.3, the three-dimensional eigenspace of $A$ corresponding to the eigenvalue $(-1)^{p}$ is spanned by $u_{\kappa, p+2}, u_{\kappa, p+4}$, and $u_{\kappa, p+6}$. The matrix of $B$ restricted to $\left\langle u_{\kappa, p+2}, u_{\kappa, p+4}, u_{\kappa, p+6}\right\rangle$ is

$$
\left(\begin{array}{ccc}
0 & 0 & -e^{2 \pi i \frac{p(p+6)}{\kappa}} \\
0 & (-1)^{p+1} & 0 \\
-e^{2 \pi i \frac{p(p+2)}{\kappa}} & 0 & 0
\end{array}\right) .
$$


Thus, $B$ has eigenvalues $(-1)^{p}$ and $(-1)^{p+1}$ of multiplicities 1 and 2 , respectively. The eigenspace corresponding to the eigenvalue $(-1)^{p}$ is spanned by the vector $u_{\kappa, p+2}+(-1)^{p+1} e^{2 \pi i p(p+2) / \kappa} u_{\kappa, p+6}$. So this vector is proportional to $c(\tau)\left(v_{1}-v_{5}\right)$. By Lemma 6.2, $c(\tau)$ must be a solution of the differential equation

$$
\begin{gathered}
\frac{\kappa}{p+1}\left(\frac{d}{d \tau} c(\tau)\right)\left(\theta_{6,1}(0, \tau)-\theta_{6,5}(0, \tau)\right)= \\
-6\left(\frac{d}{d \tau} \ln \vartheta_{1}^{\prime}(0, \tau)\right) c(\tau)\left(\theta_{6,1}(0, \tau)-\theta_{6,5}(0, \tau)\right)+10 c(\tau) \frac{d}{d \tau}\left(\theta_{6,1}(0, \tau)-\theta_{6,5}(0, \tau)\right) .
\end{gathered}
$$

The function $c(\tau)=\left((2 \pi)^{3}\left(\theta_{6,1}(0, \tau)-\theta_{6,5}(0, \tau)\right)^{5} \vartheta_{1}^{\prime}(0, \tau)^{-3}\right)^{2(p+1) / \kappa}$ is a solution of the preceding equation. By Lemma 7.5, $c(\tau)=(\eta(\tau))^{-8(p+1) / \kappa}$. The coefficient of proportionality is computed as in the proof of (2). Notice that the solution in (11) is invariant with respect to the action of the modular group.

\section{References}

[A1] K. Aomoto, Jacobi polynomials associated with Selberg integrals, SIAM J. Math. Anal., 18, (1987), 545-549.

[A2] _ On the complex Selberg integral, Q. J. Math. Oxford, 38, (1987), 385-399.

[As] R. S. Askey, Some basic hypergeometric extensions of integrals of Selberg and Andrews, SIAM J. Math., 11, (1980), 938-951.

[D] V. Dotsenko, Solving the SU(2) Conformal Field Theory with the Wakimoto FreeField Representation, Nuclear Phys. B 358 (1991) 547-570.

[DF1] V. Dotsenko and V. Fateev, Conformal algebra and multipoint correlation functions in 2-D statistical models, Nucl. Phys., 240, (1984), 312-348.

[DF2] - Four-point correlation functions and the operator algebra in 2-D conformal invariant theories with central charge $C \leq 1$, Nucl. Phys., 251, (1985), 691-734.

[EK] P. Etingof, A. Kirillov, Jr., On the affine analogue of Jack's and Macdonald's polynomials, Duke Math J. 78 (1995), 229-256.

[FSV1] G. Felder, L. Stevens, and A. Varchenko, Elliptic Selberg integrals, math.QA/0103227.

[FSV2] - Modular transformations of the elliptic hypergeometric functions, Macdonald polynomials, and the shift operator, math. QA/0203049, to appear in Moscow Math J.

[FV1] G.Felder and A.Varchenko, Integral Representations of solutions of the elliptic Knizhnik-Zamolodchikov-Bernard equations, Int. Math. Res. notices, 5, (1995), 221233.

[FV2] , The q-Deformed KZB-heat Equation, math. QA/9809139.

[HM] J. Harnad and J. McKay, Modular Solutions to Equations of Generalized Halphen Type, math. MP/9804006.

[Ma] I.G. Macdonald, Symmetric Functions and Orthogonal Polynomials, American Mathematical Society, (1998).

[M] M.L. Mehta, Random Matrices, Academic Press, (1991).

[S] A. Selberg, Bemerkninger om et multiplet integral, Norsk Mat. Tidsskr., 26, (1944), $71-78$.

[W] M. Wakimoto, Infinite-Dimensional Lie Algebras, American Mathematical Society, (1999).

[WW] E.T. Whittaker and G.N. Watson, Modern Analysis, Cambridge University Press, (1927). 
Departement Mathematik, ETH-Zentrum, 8092 Zürich, SwitZerland

E-mail address: felder@math.ethz.ch

Department of Mathematics, University of North Carolina at Chapel Hill, Chapel HiLl, NC 27599-3250, USA

E-mail address: lstevens@email.unc.edu

Department of Mathematics, University of North Carolina at Chapel Hill, Chapel HiLL, NC 27599-3250, USA

E-mail address: anv@email.unc.edu 